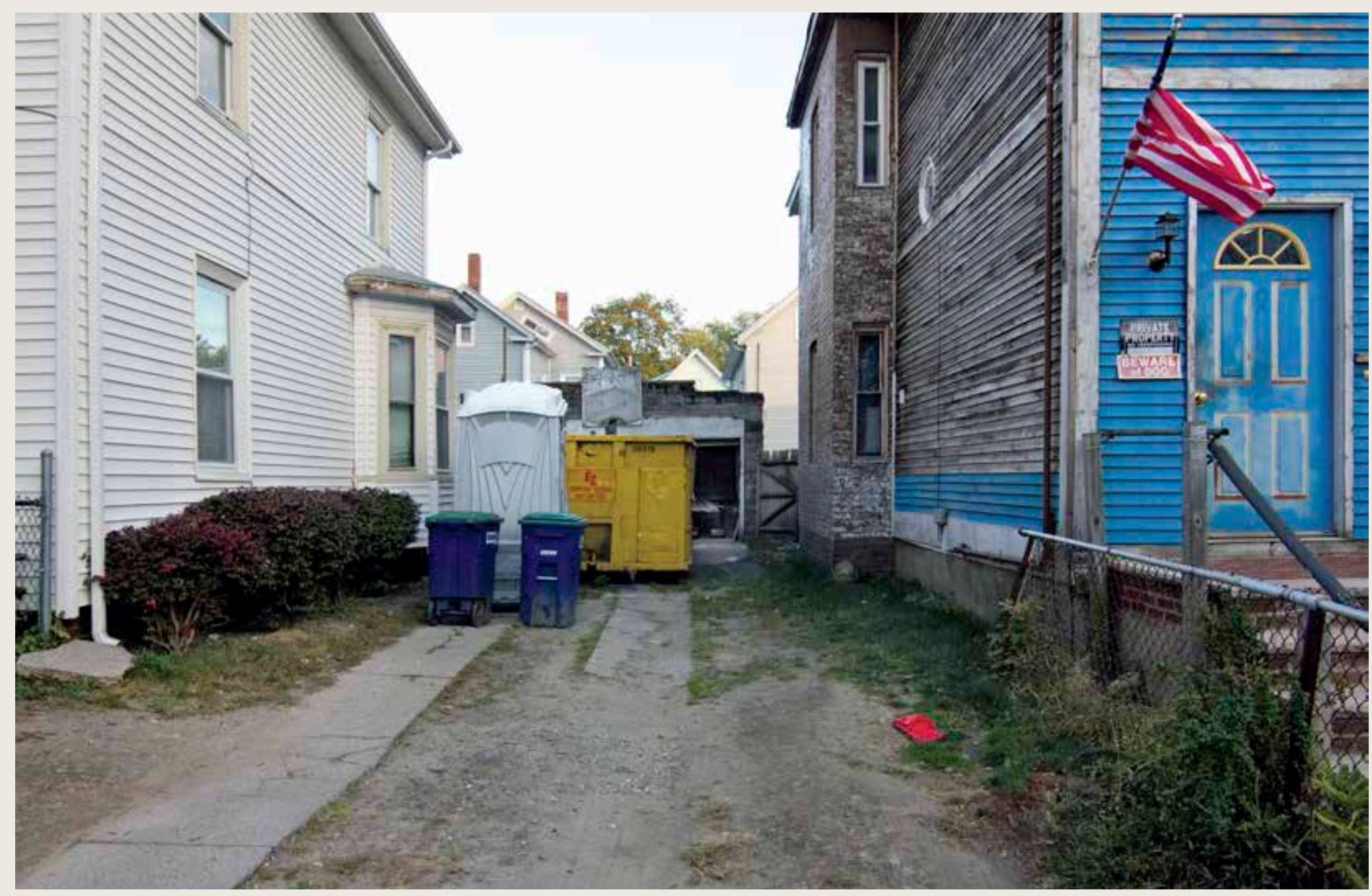

25 - 27 Quincy Street. Boston, MA. E E.UU. (c) Stephannie Fell

\title{
THE MID-SPACE
}

STEPHANIE FELL

If the line that divides two properties configures the minimum fragment of collectivity -in being the encounter of two individualities-, what can these minimal fragments say about the culture in which they exist?

The Mid-space (the 'space in between' or the 'space halfway') is a photographic essay about the space in between dwellings throughout different neighborhoods of Boston, United States. The photographs here presented aren't a view of the façade -that is, the desired portrayed image- but rather those everyday spaces we tend to overlook, where the common turns into a political position: the value of the individual, indifference, the notion of community and even silent violence confronted with the urban ideal of the American dream. ARQ 

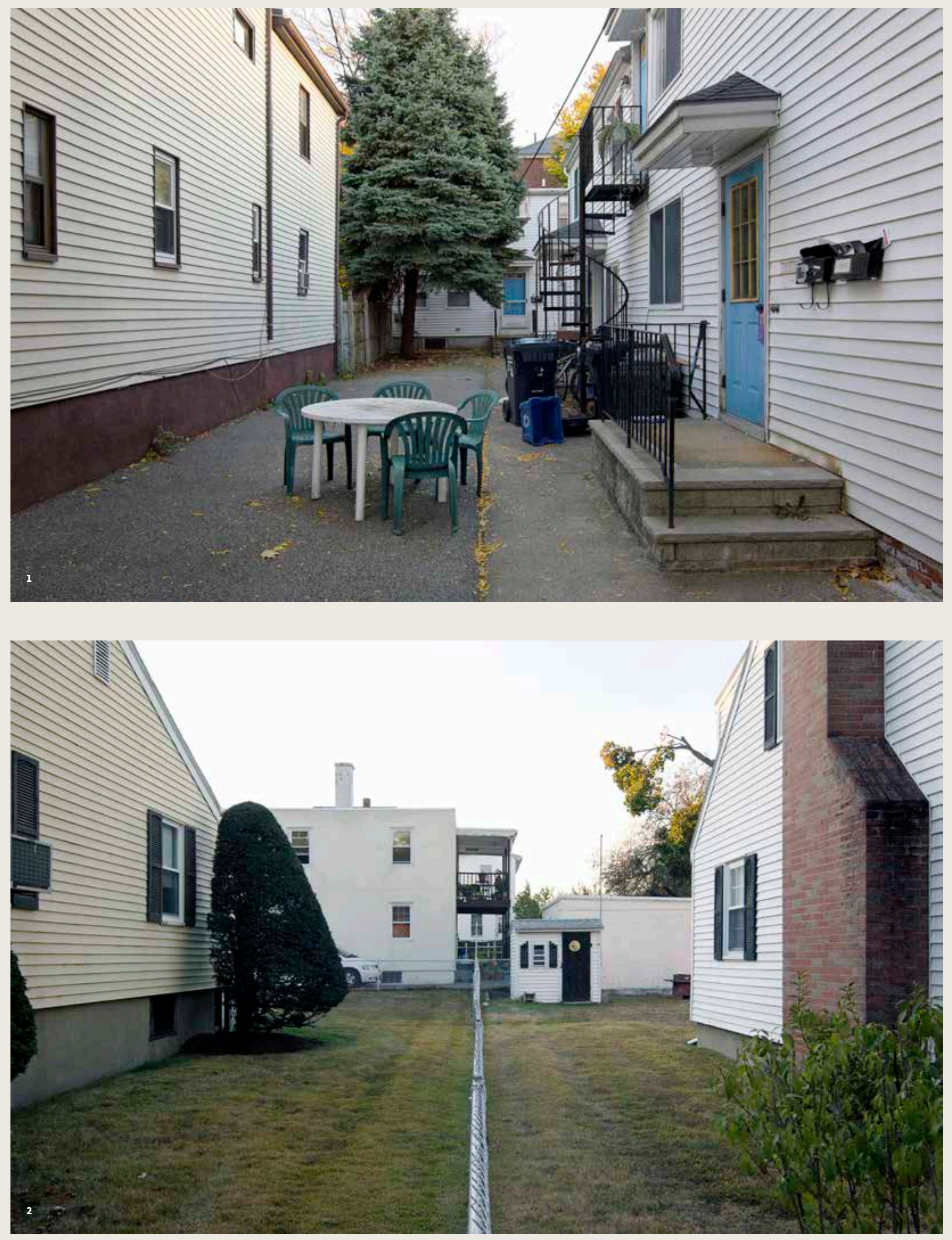


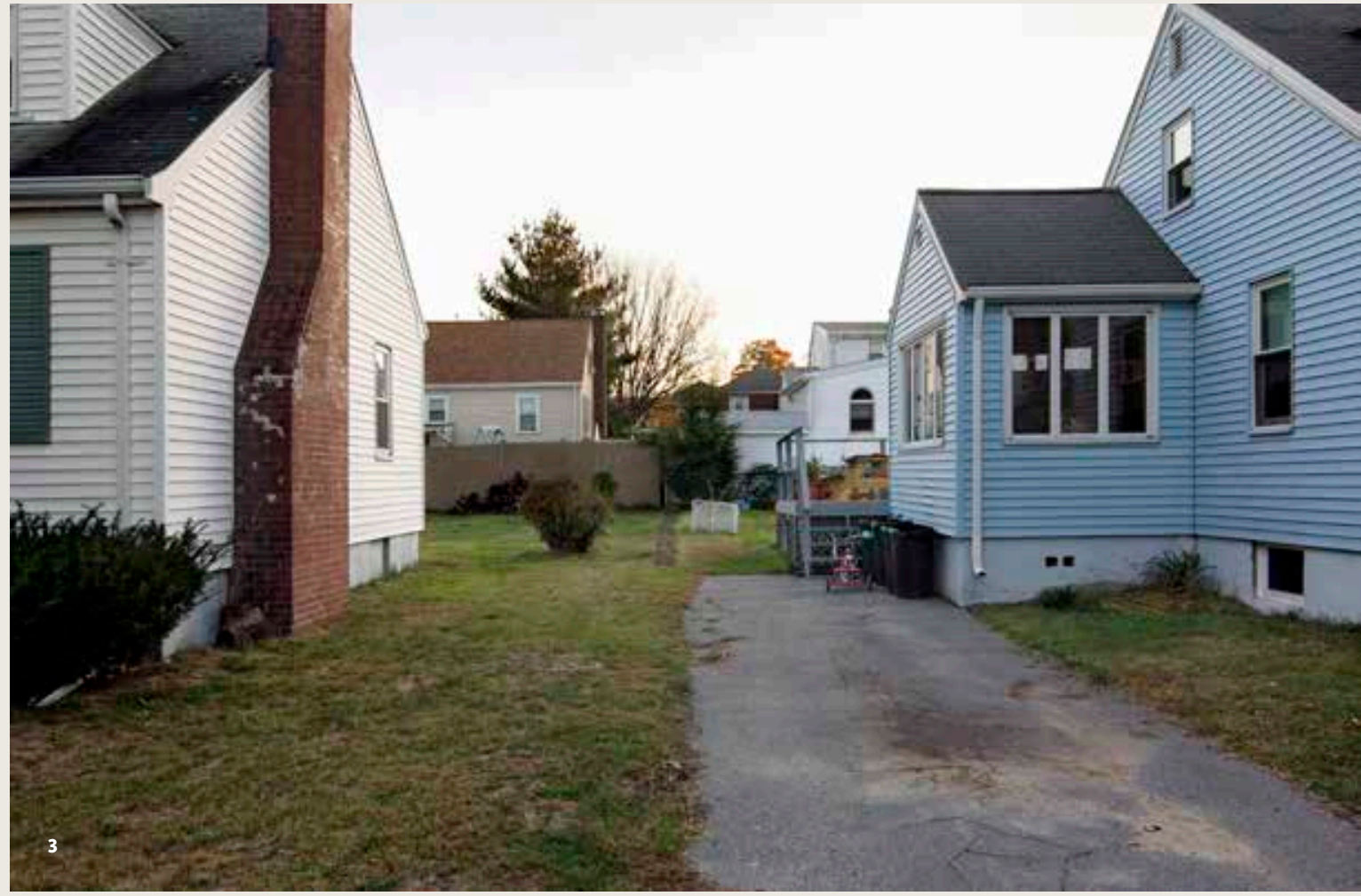

1 140-144 Thorndike Street. Boston, MA. EE.UU. (c) Stephannie Fell

STEPHAN NIE FELL

<info@stephanniefell.com>

$230-32$ River Street. Boston, MA. EE.UU. (c) Stephannie Fell

3 7-11 University Road. Boston, MA. EE.UU. c) Stephannie Fell

Architect, Pontificia Universidad Católica de Chile (2012). She studied photography at the Massachusetts College of Art and Design, Boston, USA (2013) as well as in several workshops with relevant local and international photographers. She is Professor of Architecture and Landscape Photography at the Pontificia Universidad Católica de Chile.

4 23-27 Wellesley Road. Boston, MA. EE.uU. (c) Stephannie Fell

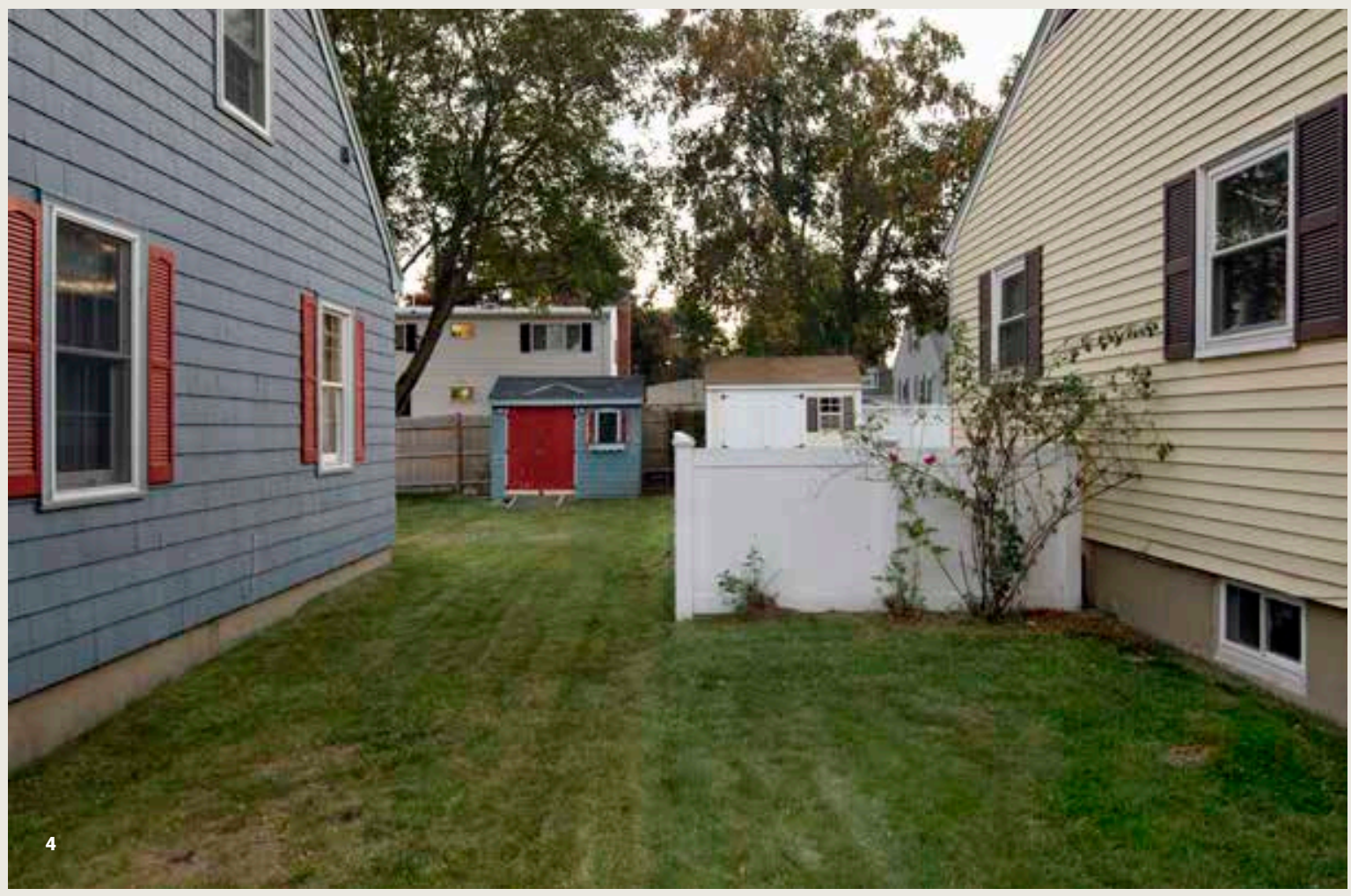

